Dierk Hoffmann

\title{
Revolution und Gegenrevolution im SED-Geschichtsverständnis. Otto Grotewohls Deutung der Novemberrevolution 1918 und des Volksaufstands 1953
}

Für die Sozialistische Einheitspartei Deutschlands (SED) stellte Geschichtspolitik von Anfang an eine wichtige "herrschaftslegitimatorische Systemstütze» dar. ${ }^{1}$ Auf diese Weise sollte in der DDR-Bevölkerung Systemloyalität erzeugt werden. ${ }^{2}$ Dabei nahm die Deutung der Weimarer Republik und der Novemberrevolution von 1918 eine zentrale Rolle ein. Denn mit der Abdankung Kaiser Wilhelm II. und dem Ende der Monarchie in Deutschland stand aus Sicht der SED die Schaffung einer sozialistischen Gesellschaftsordnung erstmals ganz oben auf der Tagesordnung. Der ostdeutsche Teilstaat inszenierte sich quasi als Lehre aus Weimar, so etwa im ersten Aufruf des Zentralkomitee (ZK) der Kommunistischen Partei Deutschlands (KPD) vom 11. Juni 1945, aber auch noch bei der letzten LiebknechtLuxemburg-Demonstration im Januar 1989. Martin Sabrow brachte dieses geschichts- und erinnerungspolitische Selbstverständnis prägnant auf den Punkt: "Das Argument Weimar bildete im Legitimationsdiskurs des SED-Staates gleichsam eine geschichtspolitische Allzweckwaffe. ${ }^{3}$ So sei mit dem Verweis auf den Vertrag von Rapallo 1922 versucht worden, das Bündnis zur Sowjetunion historisch zu legitimieren. Der vertragschließende deutsche Außenminister Walter Rathenau habe folglich einen festen Platz »im Pantheon der ostdeutschen Straßen-

1 Wolfrum, Edgar: Geschichtspolitik in der Bundesrepublik Deutschland. Der Weg zur bundesrepublikanischen Erinnerung 1948 - 1990, Darmstadt 1999, S. 36.

2 Ullrich, Sebastian: Der Weimar-Komplex. Das Scheitern der ersten deutschen Demokratie und die politische Kultur der frühen Bundesrepublik (Hamburger Beiträge zur Sozial- und Zeitgeschichte, Bd. 45), Göttingen 2009, S. 562.

3 Sabrow, Martin: Kampfplatz Weimar. DDR-Geschichtsschreibung im Konflikt von Erfahrung, Politik und Wissenschaft, in: Weimar im Widerstreit. Deutungen der ersten deutschen Republik im geteilten Deutschland, hrsg. von Heinrich August Winkler, München 2002, S. 163-184, hier S. 164. 
namen« bekommen, obwohl er für die SED geradezu idealtypisch das sogenannte Monopolkapital verkörperte.

Das Ausbleiben der sozialistischen Umwälzung nach 1919 und der Untergang der Weimarer Republik konnten anders als die Niederlage in der Novemberrevolution 1918 nicht auf das Fehlen einer marxistisch-leninistischen Partei zurückgeführt werden. Hier half der Vorwurf des Verrats, mit dem die SED-Geschichtsschreibung die Sozialdemokratische Partei Deutschlands (SPD) belegte, wobei OstBerlin auf ein bereits bestehendes Narrativ der Weimarer KPD zurückgreifen konnte. Keine einzige nennenswerte Handlung der SPD-Führung fand die Zustimmung in den "Leittexten der historischen Meistererzählung der DDR«. ${ }^{4}$ Selbst das Erstarken des gemeinsamen nationalsozialistischen Gegners konnte in der SEDSicht das "Verdammungsurteil über die Sozialdemokratie» nicht mildern. Pikanterweise war es vor allem ein ehemaliger Sozialdemokrat, der Ende der 1940er bzw. Anfang der 1950er Jahre für die SED die Aufgabe übernahm, an die Novemberrevolution von 1918 öffentlich zu erinnern. Die Rede ist von Otto Grotewohl. Der SED-Ko-Vorsitzende (1946-1954) und Ministerpräsident der Deutschen Demokratischen Republik (DDR) (1949-1964) hatte einen entscheidenden Anteil am Aufbau und Ausbau der SED-Herrschaft in der DDR. So stimmte er nicht nur dem Zusammenschluss von SPD und KPD zu, sondern segnete auch die nachfolgende innerparteiliche Verfolgung ehemaliger Sozialdemokraten sowie die damit einhergehende Transformation der SED in eine kommunistische Kaderpartei ab. Darüber hinaus gab er der politischen und sozioökonomischen Neuordnung in der Sowjetischen Besatzungszone (SBZ)/DDR sein Plazet. Im Folgenden wird erstens Grotewohls biografischer Weg in der Novemberrevolution 1918 und seine politische Sozialisation skizziert, zweitens seine Rolle bei den Feierlichkeiten zum 30. Jahrestag der Novemberrevolution 1948 beleuchtet und drittens sein Umgang mit dem Volksaufstand am 17. Juni 1953 innerhalb der SED-Führung thematisiert. $^{5}$

I.

Otto Grotewohl, 1894 in Braunschweig geboren, wurde Ende November 1914 zum Militärdienst eingezogen und kam, nachdem er zunächst als "garnisonsverwen-

\footnotetext{
$4 \quad$ Ebd.

5 Die folgenden Ausführungen beruhen im Wesentlichen auf meiner Biografie über Otto Grotewohl. Vgl. Hoffmann, Dierk: Otto Grotewohl (1894-1964). Eine politische Biographie (Quellen und Darstellungen zur Zeitgeschichte, Bd. 74), München 2009.
} 
dungsunfähig“ eingestuft wurde, ${ }^{6}$ im Mai 1915 an die Ostfront nach Galizien. Fast einen Monat nach Abschluss des deutsch-russischen Friedensvertrages von Brest-Litowsk am 3. März 1918 wurde er an die Westfront verlegt, wo er bis zum Ende des Ersten Weltkriegs blieb. Ob er Mitglied eines Arbeiter- und Soldatenrates war, wie die SED-Geschichtsschreibung später behauptete, ${ }^{7}$ lässt sich nicht mehr eindeutig klären. Heinz Voßke, der 1979 in der DDR die offizielle Grotewohl-Biografie vorlegte, mutmaßte, Grotewohl habe an den bewaffneten Kämpfen gegen Georg Maerckers Freikorpstruppen teilgenommen, ohne aber Quellenbelege anzuführen. ${ }^{8}$ Da die Besatzung Braunschweigs kampflos erfolgte, sind erhebliche Zweifel an dieser These angebracht. Voßke stützte sich bei seiner Darstellung vermutlich auf Aussagen Grotewohls aus dem Jahr 1948, in denen sich dieser als Mitglied der Roten Garde bezeichnete, auf die die einrückenden Truppen Maerckers geschossen hätten. ${ }^{9}$

Die Novemberrevolution in Braunschweig fand ohne Otto Grotewohl statt. Als Herzog Ernst August am 8. November 1918 als erster deutscher Reichsfürst abdankte und die Regierungsgeschäfte dem Arbeiter- und Soldatenrat übertrug, befand sich der 24-jährige noch als Soldat an der deutsch-niederländischen Grenze. Erst kurz vor Weihnachten kehrte Grotewohl nach Braunschweig zurück. Der Weg ins Zivilleben vollzog sich für ihn weitgehend mühelos, denn er konnte seine Anstellung bei der Allgemeinen Ortskrankenkasse (AOK), die er 1914 kurz vor seiner Einberufung zum Militärdienst angetreten hatte, wieder aufnehmen. Hier blieb er bis zum 25. November 1921, als er im Braunschweiger Landtag zum zweitjüngsten Minister der Weimarer Republik gewählt wurde.

Über Grotewohls erste politische Aktivitäten wissen wir nicht sehr viel. Sein im April 1949 ausgestelltes Mitgliedsbuch des Freien Deutschen Gewerkschaftsbundes (FDGB) enthält den Vermerk, dass er seit seinem 18. Geburtstag im Verband der deutschen Buchdrucker in Braunschweig organisiert war. ${ }^{10}$ Zuvor hatte er sich bereits in der sozialistischen Arbeiterjugend Braunschweigs einen Namen gemacht. Erste Funktionen erhielt er offensichtlich im sogenannten Jugendausschuss. 1912 trat er in die SPD ein, die er im Dezember 1918 in Richtung der Un-

6 Stiftung Archiv der Parteien und Massenorganisationen der DDR im Bundesarchiv (fortan: SAPMOBArch), Nachlass Otto Grotewohl (fortan: NY 4090), Bd. 262, Bl. 5 f., hier Bl. 5., Brief Grotewohls an Adolf Dommick vom 27.11.1914.

7 Voßke, Heinz: Otto Grotewohl. Biographischer Abriß, Berlin (Ost) 1979, S. 45.

8 Ebd., S. 47.

9 Vgl. Jodl, Markus: Amboß oder Hammer? Otto Grotewohl. Eine politische Biographie, Berlin 1997, S. 28.

10 SAPMO-BArch, NY 4090/4, Bl. 15, FDGB-Mitgliedsbuch Nr. 34338 vom April 1949. 
abhängigen Sozialdemokratischen Partei Deutschlands USPD verließ.11 Nur auf den ersten Blick rückte Grotewohl nach dem Ende des Ersten Weltkriegs politisch nach links. Zur besseren Einordnung ist ein kurzer Blick auf die Entwicklung im Freistaat notwendig. ${ }^{12}$ Zwei Faktoren sind von entscheidender Bedeutung. Erstens: Anders als im Reich kam der USPD in Braunschweig eine zentrale Rolle zu, denn sie war Regierungspartei und konnte die Mehrheitssozialdemokratische Partei Deutschlands (MSPD) bei den Landtagswahlen 1920 deutlich überrunden. Im Vergleich zu anderen Bezirken verfolgte der USPD-Bezirk Braunschweig eine verhältnismäßig gemäßigte Politik: So wurden etwa die Forderungen nach einer Bodenreform sowie nach einer Sozialisierung der Wirtschaft nicht ernsthaft weiter verfolgt. Dies hing wiederum mit der frühzeitigen Regierungsbeteiligung zusammen, die letztlich eine Radikalisierung der Partei verhinderte. Darüber hinaus sprach sich die USPD in Braunschweig frühzeitig für die Durchführung von Landtagswahlen aus. Damit deutet vieles darauf hin, dass hier die Errichtung einer Räteherrschaft nicht drohte, auch wenn sich unter den Unabhängigen zahlreiche Spartakisten befanden, die dieses Ziel erklärtermaßen anstrebten. Zweitens: Anders als in den übrigen Regionen Deutschlands, wo kommunistische Aufstände von Freikorps niedergeschlagen und neue Machtverhältnisse gewaltsam hergestellt wurden, blieb die USPD in Braunschweig an der Macht. Verlierer war die KPD, die mit ihren Plänen zur gewaltsamen Errichtung der Räteherrschaft kläglich gescheitert war und in der Folgezeit zu einer »kleinen Sekte verkümmerte«.13

Die braunschweigische USPD wurde ganz besonders von der Entwicklung der Gesamtpartei auf Reichsebene geprägt. Nach einer turbulenten Gründungs- und Aufbauphase befand sich die USPD im Frühsommer 1920 zunächst noch in einem ruhigen Fahrwasser. Die Partei bot auch nach außen ein weitgehend geschlossenes Bild: Innerparteiliche Kontroversen über das Rätesystem und den Parlamentarismus sowie die Diktatur des Proletariats entwickelten keine Sprengkraft für die USPD, sondern konnten stets entschärft werden. ${ }^{14}$ Auch wenn die heftig erörterte Frage nach einer neuen militanten Internationalen nicht geklärt werden konnte, deutete doch nichts darauf hin, dass sich diese Partei binnen kurzer Zeit spalten würde. Das änderte sich im Sommer 1920 mit den sogenannten 21 Bedingungen, mit denen die nationalen kommunistischen Parteien den Weisungen Moskaus

11 Rother, Bernd: Otto Grotewohl (1894-1964). Biographische Skizze seiner Braunschweiger Jahre (1894-1933), in: Internationale wissenschaftliche Korrespondenz zur Geschichte der deutschen Arbeiterbewegung 28 (1992), S. 523 - 533, hier S. 526.

12 Vgl. Rother, Bernd: Die Sozialdemokratie im Land Braunschweig 1918 bis 1933, Bonn 1990.

13 Ebd., S. 71.

14 Vgl. hierzu Wheeler, Robert F.: Die "21 Bedingungen« und die Spaltung der USPD im Herbst 1920. Zur Meinungsbildung der Basis, in: Vierteljahrshefte für Zeitgeschichte 23 (1975), S. 117-154, hier S. $118 \mathrm{f}$. 
unterworfen werden sollten. Sie rissen innerparteiliche Gräben auf und führten zu einer dramatischen Zerreißprobe.

Während sich in Braunschweig schon frühzeitig eine klare Niederlage der Befürworter abzeichnete, blieben die Gegner der 21 Bedingungen auf Reichsebene in der Minderheit. Auch Grotewohl schloss sich dem radikalen Lager nicht an, sondern übernahm die Mehrheitsmeinung, die sich unter den Unabhängigen in Braunschweig herausgebildet hatte. Damit wies er sich als pragmatischer, d.h. ideologisch ungebundener Politiker aus. ${ }^{15}$ Auffallend ist ferner sein Eintreten für die Einheit und die Selbständigkeit der USPD, die bis dahin ohnehin schon dezentral organisiert war. ${ }^{16}$ Obwohl er radikale Maßnahmen nicht rundweg ablehnte, überwog auch bei ihm letztlich das Unbehagen, von einer ausländischen Partei (aus Moskau) fremdgesteuert zu werden. Diese Überlegung und nicht das fehlende Klassenbewusstsein, das ihm sein DDR-Biograph Voßke unterstellte, ${ }^{17}$ führte bei ihm, wie auch bei der großen Mehrheit der USPD Braunschweig zur Ablehnung der 21 Bedingungen.

Was bleibt? Die Zeitspanne von 1916 - 1923 ist zweifellos zu kurz, um belastbare Aussagen über das biographische Gepäck nicht nur von Otto Grotewohl machen zu können. Sein kritisches, ablehnendes Verhältnis zur KPD vor 1933 liefert jedoch keinerlei Erklärungsansätze dafür, dass er sich nach 1945 unter vollkommen geänderten politischen Rahmenbedingungen vom Einheitsgegner zum Einheitsbefürworter - der Fusion von KPD und SPD - und damit zum Totengräber der Sozialdemokratie in Ostdeutschland entwickeln würde.

II.

Die Zwangsvereinigung von SPD und KPD im Frühjahr 1946 in der sowjetischen Besatzungszone (SBZ) ist ein zentrales Ereignis in der deutschen Nachkriegsgeschichte, ${ }^{18}$ das bis heute auch das Bild über Otto Grotewohl maßgeblich prägt. Kaum ein anderer deutscher Politiker spaltete die gesamtdeutsche Öffentlichkeit nach 1945 so sehr wie der gebürtige Braunschweiger. In der DDR erschien Grote-

\footnotetext{
15 So auch Jodl, Amboß oder Hammer?, S. 33.

16 Wheeler, Die »21 Bedingungen«, S. 120.

17 Voßke, Otto Grotewohl, S. 57.

18 Vgl. Bouvier, Beatrix: Ausgeschaltet! Sozialdemokraten in der sowjetischen Besatzungszone und in der DDR 1945 - 1953, Bonn 1996; Hurwitz, Harold: Demokratie und Antikommunismus in Berlin nach 1945. 4 Bde., Köln 1983-1990; Klotzbach, Kurt: Der Weg zur Staatspartei. Programmatik, praktische Politik und Organisation der deutschen Sozialdemokratie 1945 bis 1965, Berlin 1982; Auf dem Weg zur SED. Die Sozialdemokratie und die Bildung einer Einheitspartei in den Ländern der SBZ. Eine Quellenedition, hrsg. von Andreas Malycha, Bonn 1995.
} 
wohl als Politiker, der als Sozialdemokrat die Lehren aus der Geschichte gezogen habe. Sein Weg zur Vereinigung der beiden Arbeiterparteien wurde von Ost-Berlin folglich als langfristig angelegter und geradliniger Prozess dargestellt. Grotewohl hatte sich im Herbst 1945 vom Skeptiker zum Befürworter des Zusammenschlusses von SPD und KPD gewandelt. Drei Gründe waren für seinen Meinungsumschwung ausschlaggebend: Erstens verabschiedete er sich von seinen ursprünglichen Forderungen, die eine Gleichberechtigung der Ost-SPD beim Aufbau des politischen Systems in der sowjetischen Besatzungszone vorsahen, und verfolgte nur noch eine gesamtdeutsche Option. Da er von der SPD unter Kurt Schumacher keine Unterstützung bekam, spielte er auf Zeit und setzte auf einen Meinungsumschwung der drei Westmächte in der Deutschlandfrage. Zweitens konnte er sich dem wachsenden sowjetischen Druck, dem sich auch die sozialdemokratischen Funktionäre auf der mittleren und unteren Ebene ausgesetzt sahen, spätestens nach den Parlamentswahlen in Ungarn und Österreich nicht mehr entziehen. Da er einen Rückzug aus der Politik oder gar die Flucht in den Westen Deutschlands als letzte Möglichkeit für sich ausschloss, fand er sich mit den enger werdenden Handlungsspielräumen ab und begann damit, seine sozialdemokratischen Wurzeln zu kappen. Drittens lockte sicherlich auch die Aussicht, in der SED eine führende Position einzunehmen.

Für die SED-Führung war 1948 ein historisches Gedenkjahr, das mit Kundgebungen zum 100. Jahrestags des kommunistischen Manifests eröffnet wurde. Es folgte die Tagung des 2. Volkskongresses am 17./18. März 1948 in Berlin, die unter anderem an die Märzgefallenen der Revolution von 1848 erinnern sollte. Während bürgerliche und sozialdemokratische Politiker in den drei Westzonen an die parlamentarischen Traditionen der Frankfurter Nationalversammlung erinnerten, rückte die SED bewusst das Gedenken an die Märzkämpfe der Volksmassen in den Mittelpunkt. ${ }^{19}$ Die Feierlichkeiten zeigten, dass Deutschland drei Monate vor der Währungsreform erinnerungspolitisch bereits ein geteiltes Land war. Der Erste SED-Kulturtag, der mit dem 130. Geburtstag von Karl Marx zusammenfiel, war ein weiterer Höhepunkt dieses Gedenkjahres, mit dem die ostdeutsche Einheitspartei die Deutungshoheit über die Interpretation der deutschen Geschichte gewinnen wollte. Innerhalb des Parteiapparates wurden minutiös alle relevanten Gedenktage aufgelistet und ideologische Argumente für die öffentlichen Inszenierungen geliefert. ${ }^{20}$ Dabei betonte die zuständige Abteilung Kultur und Erziehung:

19 Dietrich, Gerd: Politik und Kultur in der Sowjetischen Besatzungszone Deutschlands (SBZ) 1945 1949. Mit einem Dokumentenanhang. Bern u. a. 1993, S. 117.

20 SAPMO-BArch, NY 4090/300, Bl. 2-6, Vorschlag der Abt. Kultur und Erziehung vom 24.12.1947 für Veranstaltungen im Jahre 1948. 
„Für die SED muss das ganze Jahr im Zeichen des Jubiläums stehen, das uns Gelegenheit zur Politisierung des deutschen Volkes gibt. Für uns sind besonders wichtig vor allem jene Daten und Ereignisse, die mit dem Entstehen der sozialistischen Bewegung verbunden [und] in eindrucksvollen Feiern und Kundgebungen zu würdigen [sind].«21 Auf der langen Liste der Gedenk- und Erinnerungstage standen schließlich der Matrosenaufstand in Kiel, die Revolutionsereignisse rund um den 9. November 1918 sowie die Reichskonferenz des Spartakusbundes und die KPD-Gründung.

Breiten Raum nahmen die Vorbereitungen für die Feierlichkeiten zum 30. Jahrestages der Novemberrevolution ein. Die Regieanweisungen kamen vom SEDParteivorstand, der sich auf seiner Sitzung am 28. Juli 1948 ausführlich mit dem Thema beschäftigte. Dazu hielt Otto Grotewohl ein langes Referat, das von Mitarbeitern des Parteiapparates ausgearbeitet und 1953 in überarbeiteter und erweiterter Fassung publiziert wurde. Unter dem Titel »Die November-Revolution und die Lehren aus der Geschichte der deutschen Arbeiterbewegung“ setzte er sich kritisch mit der Politik der SPD in der Weimarer Republik auseinander. ${ }^{22}$ Ähnlich wie 1918 gelte es auch dreißig Jahre später, die Einheit der Arbeiterbewegung "auf dem festen Boden des Marxismus « zu verwirklichen. ${ }^{23}$ In kommunistischer Manier geißelte er den politischen Kurs der sozialdemokratischen Parteiführung und warf insbesondere der West-SPD vor, "unter den Nachwirkungen früherer opportunistischer Irrungen" zu glauben, auf die "marxistische Grundorientierung" verzichten zu können. ${ }^{24}$ Grotewohl sah in der Verbürgerlichung der deutschen Sozialdemokratie in der Novemberrevolution die tiefere Ursache für den politischen Kurs von Kurt Schumacher und Erich Ollenhauer.

Grotewohls Referat hatte aber noch eine weitere Stoßrichtung, die sich gegen die bei der SED-Gründung vereinbarte Parität zwischen SPD und KPD richtete. Diese bestand noch auf dem Papier, obwohl sie schon vor 1948 weitgehend ausgehöhlt worden war. Grotewohl erteilte nämlich der Vorstellung eine Absage, bei der Vereinigung der beiden Arbeiterparteien müssten die ehemaligen Sozialdemokraten etwas radikaler und die ehemaligen Kommunisten etwas zahmer in ihrem Auftreten und ihren Forderungen werden. Grotewohl übernahm damit das kommunistische Vokabular, denn er verlangte von seinen früheren sozialdemokrati-

\footnotetext{
21 Ebd., Bl. 2.

22 Das Referat ist mit Auslassungen abgedruckt in: Friedrich, Thomas/ Hübner, Christa/ Mayer, Herbert/ Wolf, Kerstin (Hrsg.): Entscheidungen der SED 1948. Aus den Stenographischen Niederschriften der 10. bis 15. Tagung des Parteivorstandes der SED, Berlin 1995, S. $202-246$. Der Text wurde in der DDR leicht verändert publiziert. Vgl. Grotewohl, Otto: Dreißig Jahre später. Die Novemberrevolution und die Lehren der Geschichte der deutschen Arbeiterbewegung, Berlin (Ost) 1953.

23 Ebd., S. 9.

24 Ebd., S. 10.
} 
schen Parteifreunden in der SED eine völlige Abkehr vom »Opportunismus«. Dagegen sollten die Kommunisten in der Einheitspartei sogenannte sektiererische Tendenzen bekämpfen. Während Grotewohl in seinen Reden bis zu diesem Zeitpunkt durch eine vorsichtige Wortwahl aufgefallen war, verwendete er nun eine unmissverständliche, teilweise sogar martialische Sprache: „Wenn wir, die früheren Sozialdemokraten, den Opportunismus restlos begriffen haben und ausrotten können, und wenn die früheren kommunistischen Genossen den aus ihrer Partei stammenden Geist des Sektierertums restlos unter ihre Füße bekommen und totkriegen, dann kann auf diesem Boden die Sozialistische Einheitspartei die wirkliche Voraussetzung für eine fruchtbringende Einheit der deutschen Arbeiterbewegung schaffen. ${ }^{25}$ Mit der einseitigen historischen Analyse versuchte Grotewohl nicht nur die Zwangsvereinigung nachträglich erneut zu rechtfertigen, sondern auch den von der West-SPD vorgetragenen Vorwurf zu entkräften, er sei ein Renegat.

Die vom SED-Parteivorstand am 16. September 1948 verabschiedete Entschließung »Die Novemberrevolution und ihre Lehren für die deutsche Arbeiterbewegung « ließ keinen Zweifel am angeblichen Versagen der SPD in der Weimarer Republik. Die Reichsregierung unter der Leitung von Philipp Scheidemann und Gustav Bauer sei der Versuch gewesen, die Monarchie «mit Hilfe der sozialdemokratischen Führer entgegen den Interessen der Volksmassen« zu retten. ${ }^{26}$ Die Novemberrevolution von 1918 sei daher eine "unvollendete bürgerliche Revolution" gewesen, weil erstens eine revolutionäre Partei der Arbeiterklasse gefehlt habe und zweitens die »rechten sozialdemokratischen Führer» eine "konterrevolutionäre» Politik betrieben hätten. ${ }^{27}$

Die beiden Parteivorstandssitzungen Ende Juni und Ende Juli 1948 bildeten den Startschuss für eine beispiellose Kampagne gegen den sogenannten Sozialdemokratismus, ${ }^{28}$ zu der Grotewohl mit seiner Rede zur Novemberrevolution die ideologische Begründung geliefert hatte. Scharfe Kritik musste er sich von Gustav Klingelhöfer gefallen lassen, der die Ost-SPD kurz vor der Zwangsvereinigung verlassen hatte. In einem Privatbrief machte er Grotewohl für die Verfolgung von ehemaligen Sozialdemokraten in der sowjetischen Besatzungszone verantwort-

25 Friedrich/Hübner/Mayer/Wolf (Hrsg.), Entscheidungen der SED, S. 241. In der 1953 veröffentlichten Version ist diese Textpassage sprachlich etwas entschärft worden. Grotewohl, Dreißig Jahre später, S. 138.

26 Dokumente der Sozialistischen Einheitspartei Deutschlands. Beschlüsse und Erklärungen des Parteivorstandes des Zentralkomitees und des Politischen Büros. Bd. II, Berlin (Ost) 1952, S. 107-124, hier S. 114.

27 Ebd., S. 117.

28 Vgl. Bouvier, Ausgeschaltet!; Hurwitz, Harold: Die Stalinisierung der SED. Zum Verlust von Freiräumen und sozialdemokratischer Identität in den Vorständen 1946-1949, Opladen 1997; Malycha, Andreas: Die SED. Geschichte ihrer Stalinisierung 1946 - 1953, Paderborn 2000. 
lich und forderte eine Porträtzeichnung zurück, die er ihm 1945 geschenkt hatte: "Menschlich bedauere ich, Ihnen diese Nachricht nicht ersparen zu können; aber es geht um das Leben gutgläubiger Genossen, und ihre Verantwortung wiegt zu schwer. ${ }^{29}$ Nach dem veröffentlichten Beschluss des Parteivorstandes vom 29. Juli 1948 über die »organisatorische Festigung der Partei und [die] Säuberung von feindlichen und entarteten Elementen« zeigten sich viele Parteimitglieder tief beunruhigt. So musste das Büro Grotewohl auf Anfrage klarstellen, dass »schwarze Listen in unserer Partei überhaupt nicht geführt werden «. ${ }^{30}$ Gleichzeitig wiesen die Mitarbeiter des Ko-Vorsitzenden der SED den von einem Parteifreund geäußerten Verdacht zurück, Grotewohl stehe bereits auf einer »schwarzen Liste«. Auch im Ostbüro der SPD gab es Mutmaßungen über die politische Zukunft Grotewohls. In einem namentlich nicht gezeichneten Informationsbericht wurde behauptet, Grotewohl habe auf einer Sitzung des Zentralsekretariats Anfang 1948 eine Säuberungsaktion zunächst noch abgelehnt. ${ }^{31}$ Erst unter Druck habe er schließlich - so das Gerücht - die entsprechende Beschlussvorlage für den Parteivorstand widerspruchslos akzeptiert.

Das Verhalten anderer Sozialdemokraten im Umfeld Grotewohls sagt einiges über die Anpassungselastizität Grotewohls aus, denn zu einem sehr frühen Zeitpunkt hatten bereits einige politische Weggefährten wie Klingelhöfer oder Gustav Dahrendorf die Partei verlassen und waren in den Westen Deutschlands gegangen. Im Zuge des 1948 ausgerufenen Kampfes gegen den sogenannten Sozialdemokratismus sollten weitere Parteifreunde folgen. Prominentestes Beispiel ist Erich W. Gniffke, Grotewohls engster Freund und Berater. Anders als Grotewohl lehnte Gniffke die Ausrichtung der SED am sowjetischen Vorbild $\mathrm{ab}^{32}$ und floh Ende Oktober 1948 in die Westsektoren Berlins.

Wieso stellte sich Grotewohl an die Spitze der Kampagne und rief zum Kampf gegen seine ehemaligen Parteifreunde auf? Entscheidend waren für ihn die Einheit der Arbeiterbewegung und das Festhalten an der 1946 vollzogenen organisatorischen Vereinigung der beiden Arbeiterparteien, die er gegenüber westlichen Kritikern verteidigte. Dieses Motiv war Ausgangspunkt seiner Rede über die Novemberrevolution von 1918. Insofern stellt seine Zustimmung zur Verfolgung ehemaliger Sozialdemokraten 1948 keinen Bruch seiner politischen Leitbilder dar, sondern erscheint vielmehr in der Kontinuität zu seinem politischen Han-

29 Landesarchiv Berlin (fortan: LAB), Nachlass Gustav Klingelhöfer (fortan: E Rep. 200 - 23), Nr. 88, Klingelhöfer am 9.8.1948 an Grotewohl.

30 SAPMO-BArch, NY 4090/611, Büro Grotewohl am 29.10.1948 an R. J. (Hohndorf, Kreis Glauchau). 31 Archiv der sozialen Demokratie Bonn (fortan: AdsD), Ostbüro der SPD, Aktenordner Otto Grotewohl I, Aktennotiz vom 21.12.1949, S. 1.

32 Malycha, Die SED, S. $321 \mathrm{f}$. 
deln seit der Zwangsvereinigung 1946. Außerdem wollte er sicherlich den ehemaligen Kommunisten in der SED beweisen, dass er einen persönlichen Beitrag zur SED-Gründung geleistet und das ideologische Gedankengut des Marxismus-Leninismus übernommen hat. Auf diese Weise konnte er, so ließe sich weiter vermuten, das eigene politische Überleben langfristig absichern.

III.

In seiner Entschließung vom 16. September 1948 hatte der SED-Parteivorstand der Weimarer SPD vorgeworfen, eine angeblich konterrevolutionäre Politik betrieben zu haben. Dieser Vorwurf diente - wie bereits erwähnt - als Begründung zur Verfolgung und Inhaftierung ehemaliger Sozialdemokraten in der frühen DDR. Der Tonfall und die repressive Politik der SED verstärkten sich vor dem Hintergrund des ausbrechenden Kalten Krieges und hatten ihren Höhepunkt kurz nach dem blutig niedergeschlagenen Juniaufstand 1953. Regimegegner und innerparteiliche Feinde wurden nicht mehr nur als "Konterrevolutionäre», sondern als "Faschisten« stigmatisiert. Der Volksaufstand am 17. Juni 1953 war in den Augen der SED ein konterrevolutionärer, ja sogar ein faschistischer Putsch.

Diese Zuschreibung beruhte unter anderem auch auf dem Narrativ des von der SED propagierten Gegenbegriffs, d.h. des Antifaschismus. Dabei handelt es sich um einen Begriff, »der aus dem Selbstverständnis der kommunistischen Bewegung seit dem Beginn der zwanziger Jahre herrührt und von dort in die offizielle politische Standortbestimmung der DDR übernommen wurde. In ihm spiegelt sich eine realgeschichtliche Kontinuität der Auseinandersetzung der deutschen Linken mit der nationalsozialistischen Bewegung«. ${ }^{33}$ Er verweist gleichzeitig auf »den hohen Anteil der illegalen kommunistischen Organisationen am deutschen Widerstand". Darüber hinaus ist mit dem Begriff Antifaschismus »jenes Gefüge von ordnungspolitischen Konzepten, strukturellen Eingriffen und politisch-kulturellen Wertsetzungen bezeichnet, mit dem in der SBZ und DDR der kommunistische Anspruch einer konsequenten Abrechnung mit dem Nationalsozialismus praxisrelevant wurde». Schließlich ist der Begriff in der DDR »in hohem Maße politisch instrumentalisiert und jeweils mit ganz unterschiedlichen Inhalten unterlegt

33 Danyel, Jürgen: Die geteilte Vergangenheit. Gesellschaftliche Ausgangslagen und politische Dispositionen für den Umgang mit Nationalsozialismus und Widerstand in beiden deutschen Staaten, in: Historische DDR-Forschung. Aufsätze und Studien, hrsg. von Jürgen Kocka (Zeithistorische Studien, Bd. 1), Berlin 1993, S. 129 - 147, hier S. 133. 
worden« und diente als Abgrenzung zur parlamentarischen Demokratie der Weimarer Republik und nach 1949 der Bundesrepublik.

Der Antifaschismus-Begriff der SED hatte in der DDR eine hohe gesellschaftliche und generationelle Relevanz und diente der Herrschaftssicherung. Dabei schien es der SED anfangs zu gelingen, den von ihr inhaltlich besetzten Begriff einer teilweise "ganz anders politisch orientierten Bevölkerung zu ıverordnen «. ${ }^{34}$ Insbesondere für die 1945 jüngste Erwachsenengeneration konnte »die historische Beglaubigung der antifaschistischen Mythen ein hohes Maß an Plausibilität gewinnen, und zwar vor allem aus zwei Gründen. Zum einen erlebten nicht wenige der 15- bis Anfang 20-jährigen das Kriegsende und die Besetzung Deutschlands in besonderem Maße als Trauma», weil dadurch ihre bisherige Wirklichkeitsordnung zusammenbrach. Zum anderen wurde ihnen nach 1945 »die Chance zur Konversion zum Sozialismus geboten" und damit die Chance zum eigenen sozialen Aufstieg. Doch die antifaschistischen Narrative konnten auf die älteren und die nachwachsenden Generationen "nicht im gleichen Maße Anziehungskraft ausüben«. ${ }^{35}$ Denn für diese Alterskohorten gab es weder »die Notwendigkeit zur Konversion, noch gab es, [...], vergleichbare soziale Aufstiege«.

Der Antifaschismus-Begriff ließ sich in der DDR von Anfang an immer wieder rasch reaktivieren, so auch bei der geschichtspolitischen Deutung des Volksaufstandes vom 17. Juni 1953 durch die SED. Otto Grotewohl, wie auch die gesamte SED-Führung, scheinen von der Aufstandsbewegung zunächst völlig überrascht gewesen zu sein. ${ }^{36} \mathrm{Zu}$ diesem Zeitpunkt trat Grotewohl nur noch selten öffentlich auf: Am 15. Mai hielt er eine Begrüßungsansprache vor dem IV. Kongress der Gesellschaft für Deutsch-Sowjetische Freundschaft in Berlin, knapp einen Monat später referierte er am 16. Juni auf der Parteiaktivtagung der Ostberliner SED und am 24. Juni sprach er vor der Belegschaft des Kombinats "Otto Grotewohl» in Böhlau. Vor allem seine Rede am 16. Juni, mit der er den SED-Parteifunktionären in Ost-Berlin einen Politikwechsel (»Neuer Kurs») erklären wollte, machte seine gestiegene Unsicherheit deutlich. Dabei gestand er Fehler der Parteiführung ein und unterstrich die Bedeutung der Einheit von Partei und Bevölkerung: „Wir haben stets den Standpunkt vertreten, Volk und Regierung gehören zusammen. An diesem Standpunkt hat sich nichts geändert. Das gilt nicht nur in guten Tagen, sondern das gilt auch besonders in ernsten Tagen. ${ }^{37}$

34 Classen, Christoph: Faschismus und Antifaschismus. Die nationalsozialistische Vergangenheit im ostdeutschen Hörfunk (1945 - 1953) (Zeithistorische Studien, Bd. 27), Köln/ Weimar/ Wien 2004, S. 315. 35 Ebd., S. 316.

36 Für das Frühjahr und den Sommer 1953 liegen so gut wie keine Selbstzeugnisse Grotewohls vor; sein Terminkalender weist sogar für die erste Jahreshälfte 1953 erhebliche Lücken auf.

37 SAPMO-BArch, NY 4090/177, Bl. 272 - 284, hier Bl. 272, Manuskript der Rede Grotewohls vom 16.6. 1953. 
Im Vorfeld hatte die SED-Führung von der Hauptverwaltung Deutsche Volkspolizei und vom Ministerium für Staatssicherheit genügend Berichte erhalten, die deutlich machten, dass sich die DDR ungefähr ab Ende 1952 in einer schweren wirtschaftlichen und politischen Krise befand. Der beschleunigte Ausbau der Schwerindustrie unter Vernachlässigung der Konsumgüterindustrie, Steuerungsprobleme der sich etablierenden Planwirtschaft, die einsetzende Kollektivierung der Landwirtschaft, die rasant ansteigenden Zahlen von sogenannten Republikflüchtigen sowie Haushaltsdefizite durch den Aufbau bewaffneter Organe hatten letztlich zu dieser Krise geführt. Die Polizei- bzw. Stasi-Berichte enthielten darüber hinaus aber auch deutliche Hinweise auf eine drastische Verschlechterung der Stimmungslage in der Bevölkerung. Die Akzeptanz des politischen Systems und besonders der SED-Herrschaft in weiten Teilen der Bevölkerung schien bedrohlich zu bröckeln. Gleichwohl zogen die Politbüromitglieder keine direkten Konsequenzen aus dieser prekären innenpolitischen Lage. Der Anstoß zu einem Kurswechsel erfolgte von außen, durch Moskau. Am 2. Juni reiste die SED-Führung in die sowjetische Hauptstadt und erhielt dort von der neuen politischen Führung - Stalin war am 5. März 1953 gestorben - die Anweisung für den »Neuen Kurs«.38 Für das Politbüro der SED bestand ein gewaltiger Handlungsdruck: So mussten die Funktionäre sowie die Basis der Partei von der Notwendigkeit überzeugt werden, den seit der II. Parteikonferenz 1952 eingeschlagenen politischen Kurs abzubrechen. Des Weiteren mussten Maßnahmen ergriffen werden, um die sich dramatisch verschlechternde Versorgungslage der Bevölkerung zumindest zu stabilisieren und mittelfristig zu verbessern. Die Tatsache, dass die umstrittene Normenerhöhung aber nicht revidiert wurde, was dann zum Ausbruch des Volksaufstandes führte, verdeutlichte das mangelhafte Krisenmanagement des SED-Politbüros.

Die SED-Führung wirkte im entscheidenden Moment wie paralysiert und tauchte zunächst einmal ab. Sie begab sich während des Volksaufstands in die Obhut der Besatzungsmacht nach Berlin-Karlshorst, die nun das Heft des Handelns an sich riss. Die Befehlshaber der sowjetischen Truppen verhängten noch am 17. Juni den Ausnahmezustand und ließen Panzer in den Straßen auffahren. Die Erhebung gegen das SED-Regime wurde regelrecht niedergewalzt. Es gab viele Tote und Verletzte. ${ }^{39}$ Die ostdeutsche Justiz war noch lange Zeit mit der straf-

38 Anweisungen der sowjetischen Führung »Über Maßnahmen zur Gesundung der politischen Lage in der Deutschen Demokratischen Republik« (o.D.), in: Die DDR vor dem Mauerbau. Dokumente zur Geschichte des anderen deutschen Staates 1949-1961, hrsg. von Hoffmann, Dierk/ Schmidt, Karl-Heinz/ Skyba, Peter, München/Zürich 1993, S. $152-158$.

39 Die Toten des Volksaufstandes vom 17. Juni 1953, hrsg. von Ahrberg, Edda/ Hertle, Hans-Hermann/ Hollitzer, Tobias, Münster 2004. 
rechtlichen Verfolgung der vermeintlichen Rädelsführer beschäftigt; eine große Verhaftungswelle überzog die DDR. Nachdem Ulbricht, Grotewohl und Pieck in der Parteizentrale wieder aufgetaucht waren, begann für sie der Kampf um die Deutungshoheit: Sie schoben dem Westen die Schuld für die blutigen Ereignisse in die Schuhe. Dem Volksaufstand drückten sie den Stempel "faschistischer Putsch» auf und entledigten sich so der eigenen politischen Verantwortung. So hatte der DDR-Präsident und Ko-Vorsitzende der Partei, Wilhelm Pieck, schon am 2. Juli im ıNeuen Deutschland von einem "faschistischen Abenteuer" gesprochen, das von westlichen Agenten, »Überreste[n] jener Nazis und SS-Banditen, die nicht umgelernt haben, sowie vor allem deklassierter Elemente aus Westberlin« inszeniert worden sei. ${ }^{40}$ Und Walter Ulbricht, Erster Sekretär des ZK der SED, rief auf einer ZK-Tagung Mitte September dazu auf, »die faschistischen Untergrundorganisationen, die von den verschiedenen in Westdeutschland und in Westberlin bestehenden feindlichen Agenturen organisiert werden, zu zerschlagen «. ${ }^{41}$ Das Ministerium für Staatssicherheit (MfS) startete eine umfangreiche Untersuchung, bei der vor allem angebliche Verbindungen zu westlichen Agentennetzen aufgedeckt werden sollten. Während das Politbüro in seinem Kommuniqué vom 9. Juni noch betont hatte, dass zurückkehrende "Republikflüchtige» nicht benachteiligt werden dürften, wurde Monate später eine härtere Gangart eingeschlagen: Alle zurückkehrenden Personen sollten erfasst und durch Geheime Informanten (GI) des MfS beobachtet werden. ${ }^{42}$ Die eingeleiteten Sicherheitsmaßnahmen zeigten jedoch nur begrenzte Wirkung, da es auch Wochen nach dem niedergeschlagenen Aufstand vereinzelt zu Arbeitsniederlegungen in den Betrieben kam.

Der 17. Juni 1953 war ein Schlüsselereignis in der DDR-Geschichte. Die gewaltsame Niederschlagung durch die sowjetischen Besatzungstruppen hatte erhebliche Auswirkungen auf die innenpolitische Entwicklung des Landes und löste einen Schock- und Lerneffekt sowohl bei den politisch Verantwortlichen in OstBerlin als auch bei der ostdeutschen Bevölkerung aus. Zu den kurzfristigen Folgen zählte ein Anstieg der Flüchtlingszahlen. Nach Angaben der Zentralverwaltung für Statistik verließen bis Ende 1953 knapp 300000 Menschen ihre Heimat in Richtung Westen. ${ }^{43}$ Das bundesdeutsche Notaufnahmeverfahren wies für die-

40 Wiederabdruck in: Pieck, Wilhelm: Reden und Aufsätze. Bd. III: Auswahl aus den Jahren 19501953, Berlin (Ost) 1954, S. 620 - 627, hier S. 623 (Zitat).

41 Ulbricht, Walter: Die Entwicklung des deutschen volksdemokratischen Staates 1945 - 1958, Berlin (Ost) 1961, S. $332-334$, hier S. 333 f. (Zitat).

42 Vgl. Dienstanweisung Nr. 1/54 der BVfS Cottbus vom 5.1.1954, in: Freiheit wollen wir! Der 17. Juni 1953 in Brandenburg, hrsg. von Burghard Ciesla, Berlin 2003, S. 210 - 212.

43 »Republikflucht«. Flucht und Abwanderung aus der SBZ/DDR 1945 bis 1961, hrsg. von Melis, Damian van/ Bispinck, Henrik, München 2006, S. 255 (Tabelle 1). 
ses Jahr sogar über 330000 Zuzüge aus der DDR und Ost-Berlin aus. ${ }^{44}$ Die Abwanderung mit den Füßen war ein Zeichen für den zunehmenden Legitimationsverlust, den die SED durch die Juniereignisse erlitten hatte. Die langfristigen Folgen waren gewaltig, da der Verlauf des Volksaufstandes deutlich machte, wo sich das politische Machtzentrum eigentlich befand. Die SED-Herrschaft hatte nur aufgrund der sowjetischen Garantieerklärung weiter Bestand. Ulbricht, Pieck und Grotewohl wurden wieder einmal bewusst, dass ihre Führungsrolle von der Unterstützung im Kreml abhing. Gleichzeitig setzte sich bei großen Teilen der Bevölkerung die Einsicht durch, dass ein erneutes Aufbegehren gegen das SED-Regime sinnlos war, solange Moskau die schützende Hand über die SED-Führung hielt.

Bei der Charakterisierung des Volksaufstands vom 17. Juni als »faschistischer Putschversuch»,45 der vom Westen gelenkt worden sei, ${ }^{46}$ übernahm Grotewohl die vom SED-Politbüro gewählte Sprachregelung. Gleichzeitig ignorierte er die Tatsache, dass sich zahlreiche Arbeiter gegen das SED-Regime erhoben und damit der Hegemonialpartei das Vertrauen entzogen hatten: „Das alles zeigt, dass von einem ১berechtigten Streikı am 17. Juni keine Rede sein kann. Der 17. Juni 1953 war ein versuchter Staatsstreich, war ein faschistischer Putsch und eine Kriegsprovokation. «47 Stattdessen unterschied er zwischen der Masse der Arbeiter und den vermeintlichen Rädelsführern, die hart bestraft werden sollten: „Wir denken nicht daran, die Werktätigen, die sich irreführen und verblenden ließen, zu bestrafen oder an ihnen Rache zu nehmen. Wir werden mit allen ehrlichen und klassenbewussten Arbeitern sprechen und diskutieren, nicht aber mit Hetzern und Provokateuren. Für dieses Gesindel, das unseren friedlichen Aufbau stört, gibt es nur eins: Wir werden dafür sorgen, dass sie in Zukunft keinen Schaden mehr anrichten können." Entsprechende Anweisungen gab er auch an die ostdeutsche Presse und den Rundfunk, denn es sollte unter allen Umständen verhindert werden, dass in der DDR-Bevölkerung der Eindruck entstand, als würden die demonstrierenden Arbeiter mit den "faschistischen Provokateuren" 48 auf eine Stufe gestellt. Auch gegenüber ostdeutschen Bürgern, die sich mit Eingaben an

44 Heidemeyer, Helge: Flucht und Zuwanderung aus der SBZ/DDR 1945/49-1961. Die Flüchtlingspolitik der Bundesrepublik Deutschland bis zum Bau der Berliner Mauer, Düsseldorf 1994, S. 45 (Tabelle 4). 45 SAPMO-BArch, NY 4090/179, Bl. 2-144, hier Bl. 54, Manuskript der Rede Grotewohls auf dem 15. ZK-Plenum der SED (24.-26. 7.1953). Die Rede ist abgedruckt in: Grotewohl, Otto: Im Kampf um die einige Deutsche Demokratische Republik. Reden und Aufsätze. Bd. III, Berlin (Ost) 1959, S. 358 - 407, hier S. 378.

46 Die westliche Einflussnahme betonte Grotewohl bereits während der Nachtsitzung des ZK der SED am 21.6.1953. Vgl. SAPMO-BArch, DY 30/IV 2/1/117, Bl. 3-28, hier Bl. 13, Stenographische Niederschrift der 14. Tagung des ZK am 21.6.1953. Im weiteren Verlauf seiner Rede bedankte er sich auch noch bei den sowjetischen Besatzungstruppen für die gewaltsame Niederschlagung des Aufstandes. Ebd., Bl. 19. 47 Rede Grotewohls auf dem 15. ZK-Plenum der SED (24.-26. 7.1953), in: Grotewohl, Im Kampf um die einige Deutsche Demokratische Republik. Bd. III, S. 358-407, hier S. 388.

48 SAPMO-BArch, NY 4090/435, B1. 57, Büro Grotewohl am 23.6.1953 an Axen. 
ihn wandten, wählte er eine unmissverständliche Sprache, die keine Anzeichen von Selbstzweifeln oder Nachdenklichkeit erkennen ließ: „Wer den faschistischen Ausschreitungen westberliner Gangster und Rowdies miterlebt hat, ist den sowjetischen Offizieren und Soldaten sehr dankbar, dass sie uns vor weiteren Verbrechen bewahrt und den vom Westen zu uns geschleuderten Kriegsfunken ausgetreten haben. ${ }^{49}$

Die Zementierung der Herrschaft Ulbrichts nach dem 17. Juni engte Grotewohls Handlungsspielraum weiter ein. 1954 wurde das Amt des SED-Vorsitzenden abgeschafft. ${ }^{50}$ Damit verlor Grotewohl, der sich diese Funktion mit Pieck geteilt hatte, seine Führungsposition in der SED, die er jedoch schon zuvor de facto nicht mehr besessen hatte. Erst im Verlauf der Entstalinisierung nach dem XX. Parteitag der Kommunistischen Partei der Sowjetunion (KPdSU) 1956 bot sich erneut eine Chance, den Herrschaftsradius Ulbrichts doch noch einzuengen. Aufschlussreich ist die Reaktion des DDR-Ministerpräsidenten auf die von Chruschtschow eingeleitete Abrechnung mit Stalin. Während er noch in seinem persönlichen Tagebuch das Begräbnis Stalins 1953 melancholisch und voller Ehrfurcht vor dem Diktator schilderte, zeigen seine Aufzeichnungen drei Jahre später die Orientierungslosigkeit und Ratlosigkeit, in der er sich wie auch andere aus der SED-Führung ohne Zweifel befand:

"Jedenfalls, jetzt ist es vor allem klar: es gab keine Aera Stalin. Unbegrenzte Alleinherrschaft zerstörte in 20 Jahren die Grundsätze der Lenin'schen Partei, beseitigte jedes kollektive Handeln und jede kollektive Verantwortung. An ihre Stelle drängten sich solche Ehrgeizlinge, wie der schmachwürdige Beria, dessen Schuftereien [sic] alles und alles bis an den Rand eines furchtbaren Abgrundes zerrten. Erst dieser Parteitag hat mir klar gemacht, welche verantwortliche und ernste Arbeit die Genossen des Präsidiums der Partei in knapp 3 Jahren für die Sache des Sozialismus geleistet haben. Ich vermag selbstverständlich nur schwer ein wirklich abschließendes Urteil über das Wirken Stalins abzugeben, aber sicher ist, da[ß] schon heute über seinem Wirken die Worte stehen: ssic mundis transit gloria! [ [So vergeht der Rum der Welt!] «51

IV.

1989 tauchte das Gespenst des Volksaufstands in der DDR wieder auf: Ende August 1989 stellte der Minister für Staatssicherheit Erich Mielke bei einer internen

49 SAPMO-BArch, NY 4090/611, Büro Grotewohl am 21.6.1953 an Hertha J. (Berlin).

50 Vgl. Protokoll der Verhandlungen des IV. Parteitages der SED (30.3.-6. 4.1954). Berlin (Ost) 1954, Bd. 2, S. 947. Vgl. Amos, Heike: Politik und Organisation der SED-Zentrale 1949-1963. Struktur und Arbeitsweise von Politbüro, Sekretariat, Zentralkomitee und ZK-Apparat, Münster/ Hamburg/ London 2003, S. 320.

51 SAPMO-BArch, NY 4090/9, Bl. 865-867, Tagebuchaufzeichnungen Otto Grotewohls für seine Frau Johanna (Eintrag zum 16.02.1956). 
Leitungsbesprechung die Frage, ob es so sei, »dass morgen der 17. Juni ausbricht». Darauf gab der Geraer Bezirkschef Dieter Dangrieß selbstbewusst zu Protokoll: "Der ist morgen nicht, der wird nicht stattfinden, dafür sind wir ja auch da.» ${ }^{52}$ Am 4. Oktober erlebten rund 20000 Bürger am Dresdner Hauptbahnhof den brutalen Einsatz der DDR-Sicherheitsbehörden. Gleiches wiederholte sich am 7. und 8. Oktober in Ost-Berlin. Einen Wendepunkt markierte die Montagsdemonstration am 9. Oktober in Leipzig, an der 70000 Menschen teilnahmen und gegen die der SED-Staat erstmals nicht mehr gewaltsam vorging. Die Gewaltbereitschaft der SED-Führung war offenbar schlagartig verloren gegangen. Kurz zuvor hatte der Leipziger SED-Bezirkssekretär Kurt Meyer noch getönt: „Die Konterrevolution ist auf der Straße. Mit ihr ist ein für alle mal Schluss zu machen. - Das heißt ganz eindeutig: Gewalt anwenden."53 Am Ende blieb der Schießbefehl unausgesprochen; die Staatsmacht wich vor dem Druck der Straße zurück. Viele SED-Funktionäre waren hin- und hergerissen zwischen der Gewaltorientierung der kommunistischen Bewegung, aus der sie kamen, und dem »Friedlichkeitszwang der aktuellen Umstände». ${ }^{54}$ So erklärte der DDR-Innenminister Friedrich Dickel am 21. Oktober bei einer Dienstbesprechung: "Ich würde am liebsten hingehen und diese Halunken zusammenschlagen, dass ihnen keine Jacke mehr passt. Ich war 1953 verantwortlich hier in Berlin. [...] Mir braucht keiner zu sagen, wie man mit dem Klassenfeind umgeht. «55

Dass sich im Herbst 1989 die Ereignisse von 1953 nicht wiederholten, wird oft mit dem besonnenen Verhalten der Sowjetunion in Verbindung gebracht. Sicherlich: Ost-Berlin hatte den politischen Rückhalt Moskaus verloren - trotz der Teilnahme Gorbatschows an den Feierlichkeiten zum 40. Jahrestag der DDR-Gründung. Gorbatschow hatte der DDR als Ergebnis von Glasnost und Perestroika die Bestandsgarantie entzogen. Das erklärt allerdings nicht den Gewaltverzicht der SED am 9. Oktober 1989. Zwei Gründe waren ausschlaggebend. Erstens: Das Argument, die SED sei die Vollstreckerin der Novemberrevolution von 1918, hatte schon längst an Überzeugungs- und Bindekraft verloren. In der Ära Honecker wurde stattdessen die »Einheit von Wirtschafts- und Sozialpolitik» als Zielvorstellung propagiert und öffentlich immer wieder beschworen. Zweitens hatte sich offenbar auch in der Gerontokratie des real existierenden DDR-Sozialismus ein gewisser Wertewandel vollzogen. Demzufolge hatte die SED-Führung "Gewalt als

52 Dienstbesprechung beim Minister für Staatssicherheit (Auszug) vom 31.8.1989, in: Ich liebe euch doch alle! Befehle und Lageberichte des MfS Januar-November 1989, hrsg. von Armin Mitter und Stefan Wolle, Berlin 1990, S. 113 - 138, hier S. 125.

53 Sabrow, Martin: "1989« und die Rolle der Gewalt in Ostdeutschland, in: 1989 und die Rolle der Gewalt, hrsg. von Martin Sabrow, Göttingen 2012, S. 9-31, hier S. 15.

54 Ebd., S. 17.

55 Ebd. 
legitime[s] Mittel politischen Handelns in der sozialistischen Gesellschaft« (M. Sabrow) trotz aller Rhetorik verworfen. Oder in den Worten von Erich Mielke Ende April 1989: „Wo noch etwas mehr revolutionäre Zeiten waren, da war es nicht so schlimm. Aber jetzt, nachdem alles so neue Zeiten sind, muss man den neuen Zeiten Rechnung tragen. «56

56 Zitiert nach: Kowalczuk, Ilko-Sascha: Endspiel. Die Revolution von 1989 in der DDR, München 2009, S. 304. 
